\title{
Plasma Collection Width Measurements in a 10-cm Ring Cusp Discharge Chamber
}

\author{
Aimee A. Hubble ${ }^{1}$ and John E. Foster ${ }^{2}$ \\ University of Michigan, Ann Arbor, Michigan, 48109
}

\begin{abstract}
Effective loss area is a key parameter in determining ion thruster discharge performance and stability. A $20 \mathrm{~cm}$ partial conic ion thruster discharge chamber was designed and constructed to investigate plasma collection at the anode in small thrusters. Discharge plasma conditions and discharge performance was characterized. Effective loss area was assessed by measuring plasma current density profiles across magnetic cusps as well as measuring the widths of the dark "burn lines" that formed along the centers of the magnets. Current density profiles were obtained at different cusp probe bias voltages to contrast the relative current contributions from plasma electrons, primary electrons, and ions. Loss widths appear to scale with the hybrid radius both at the anode and at various heights above the anode. Additionally, it was found that current entering the cusp region was significantly attenuated as it is traveled toward the anode surface. This finding is consistent with the collection loss cone associated with the magnetic cusp. Also observed were significant asymmetries in current density profiles acquired across the magnetic cusps when the discharge was operated with zero gas flow. At these virtually plasma-free conditions, current profiles over cusps suggest primary electron current density is spatially extended on the side of the cusp facing the filament cathode.
\end{abstract}

\section{Introduction}

$\mathrm{T}$ HE efficiency of an ion thruster discharge is intimately related to the magnetic circuit. The magnetic circuit, typically a series of magnetic rings of alternating polarity, is used to establish a magnetic topology that increases the containment path length of the plasma particles, particularly primary electrons. The goal of the magnetic circuit design is to optimize this length so that the primary electrons as well as the sufficiently energetic electrons in the tail of the electron energy distribution function undergo at least one ionizing collision before being collected at the anode. The magnetic field imposes constraints on the electron's trajectory, thereby increasing its utilization path length. The plasma formed in the discharge chamber is lost to an effective anode surface that is determined by the magnetic circuit. In ring cusp geometries, the electrons and a significant fraction of the ions are lost at the magnetic cusps. Cross-field diffusion to anode surfaces between magnetic cusps is significantly reduced in contrast to plasma losses at the magnetic cusps, where the majority of plasma losses actually occur.

Many studies over the past 30 years or so have investigated the magnetic cusps ${ }^{1,2,3,4,5}$. These efforts were largely aimed at understanding the so-called loss width of the magnetic cusp. The loss width is literally the width of the line of collection area established by the magnetic ring. The line passes along the inner circumference of the magnetic ring and is typically centered in the middle of the magnet ring. The designation of these regions as the effective loss area is consistent with current collection measurements in ion thrusters. Here it has been observed that over 90 percent of the discharge current can be accounted for as current collected at the magnetic cusps ${ }^{6,14}$. Returning back to past studies, it was found that the loss half-width at the anode corresponded to twice the hybrid radius for low beta plasmas. Here, the hybrid radius is defined as $\sqrt{r_{e} \cdot r_{i}}$, where $\mathrm{r}_{\mathrm{i}}$ is the electron Larmor radius and $\mathrm{r}_{\mathrm{e}}$ is the ion Larmor radius ${ }^{7}$. The key unresolved question, however, is the following: Does the collection width at the anode surface determine the true effective loss area? One can visualize the magnetic cusp as a funnel similar in many respects to the phenomological picture put forth by Hershkowitz ${ }^{8}$. The funnel opening can be thought of as an

\footnotetext{
${ }^{1}$ Research Assistant, Department of Nuclear Engineering and Radiological Sciences, 2355 Bonisteel Blvd., AIAA Member.

${ }^{2}$ Associate Professor, Department of Nuclear Engineering and Radiological Sciences, 2355 Bonisteel Blvd., AIAA Senior Member.
} 
extension of the cusp into the boundary of the plasma. Here, at the mouth of the funnel, the discharge is relatively field-free and the plasma is no longer appreciably magnetized. At the very end of the funnel stem is the anode or magnet surface. The size of the opening at the anode is presumably some factor of the hybrid radius ${ }^{7,9}$. Based on the funnel model, the actual loss area for charged particles leaking from the bulk discharge should be a function of the area defined by the opening of the funnel. This is to say, one cannot simply multiply the plasma current density present at the edge of the bulk plasma by the area associated with the hybrid width to obtain plasma losses. Significant channeling and mirror action can occur as plasma enters and propagates through the funnel-like opening of the magnetic cusp, giving rise to an effective transmission coefficient that is related to the particle's position in phase space. Therefore, the loss width at the anode is not meaningful if the current density at the anode is not known. Collection loss widths are also species dependent.

Loss area to the walls or to the anode is important in that it determines both particle and energy losses. This aspect emphasizes the importance of the magnetic circuit: minimizing loss area but not without compromise to discharge stability. If one could determine the loss area for the plasma particles then one could predict not only plasma potential, but also plasma density and electron temperature as a function of discharge power and pressure ${ }^{10}$. In this case, simple particle conservation and energy conservation can be used in a relatively straightforward manner to obtain these parameters. With this information, discharge efficiency and plasma uniformity can be determined. This can all be inferred from the aforementioned data without the need for particle tracking. The specific details regarding the complicated trajectories that lead the electrons and ions to he wall is not necessary, rather the effective collection area is all that is required. To be meaningful, this effective collection area must take into account the transmission efficiency of the cusp. It is envisioned that this approach can simplify the analysis and design of ion sources for thruster applications. In this regard, the approach of this research effort is to first map the variations in the shape of the collection widths in the magnetic cusps as a function of operating conditions, compare the widths with calculated values such as $r_{e}, r_{i}$, the hybrid radius, and the burn lines formed on the cusp surface after running the source; model the widths based on local transport equations along with both measured and applied magnetic fields; and finally use the determined loss areas in conjunction with a zeroth order model to predict discharge plasma conditions and associated discharge efficiency. The final phase of this effort entails comparing the calculated discharge parameters with actual measurements and formulating an approach involving loss area for the calculation of discharge parameters. In principle, the end goal of the effort is to allow for discharge chamber design utilizing the magnetic circuit data. From the magnetic circuit, loss area can be inferred which in turn will allow for the computation of discharge conditions and efficiency. In this respect, by numerically moving magnet rings around or adding rings here and there, one can quickly optimize expected discharge performance in a straightforward fashion.

\section{A. Past research}

Plasma collection at magnetic cusps has been of interest to scientists for some time now, dating back to the early magnetic fusion days. In the early $60 \mathrm{~s}$, the so-called minimum B geometries were proposed. The notable Ioffe bar ${ }^{11}$ configuration was touted as a possible fusion geometry. In this geometry, the inner volume was relatively field-free. Plasma losses could only occur by transport through the cusps. For sufficiently narrow cusp loss widths, radial confinement times could exceed other linear confinement approaches such as the magnetic mirror. End losses, however, eventually led to the abandonment of these concepts in favor for closed geometries. In an effort to exploit plasma confinement using the magnetic cusp, Sadowsky proposed the use of a spherical source with point magnetic cusps $^{12}$. This geometry demonstrated longer confinement times than the Ioffe bar approaches, but it too was eventually abandoned in favor of the much higher performing Tokamak. Plasma source researchers, however, using the work of Sadowsky as a basis, began to experiment with the point magnetic cusp approach using permanent magnets rather than electromagnets to increase the primary electron containment length in DC ion sources. Limpaecher and McKenzie demonstrated the benefits of point cusp geometry in the improvement of not only ion source plasma density but also plasma uniformity ${ }^{13}$. Since that time, advances in permanent magnet configurations followed, leading finally to the ring cusp geometry used in present-day ion thrusters ${ }^{14}$. In order to ascertain the mechanism for increased confinement time, researchers focused their attention on the so-called loss width of the magnetic cusp. This loss width is related to the effective loss area that the magnetic cusp projects to the plasma. Hershkowitz and others found this width not to be the electron Larmor radius, but rather the so-called hybrid radius ${ }^{1}$. A theoretical basis for this measured width was put forth by Koch and Matthieussent ${ }^{9}$. They asserted that charge separation due to differences in Larmor orbits gives rise to an electric field that tends to confine the ions so that the total collection width is larger than the electron Larmor radius but smaller than the ion Larmor radius. A number of additional studies regarding the measurement of cusp widths have been carried out on magnetic cusps comprised of both electromagnets and permanent magnets. Horiike and colleagues made cusp width measurements in a filament 
source using thermocouple sweeps ${ }^{15}$. Here the heat transport to the thermocouple was assumed to be proportional to the charge flow. Arakawa and Hamatani also conducted similar sweeps, but this time with an electrostatic probe ${ }^{16}$. Close sweeps in both efforts revealed loss widths close to the hybrid radius. The work presented herein differs from previous work in that here, a map of the collection cusp region is obtained and studied. In addition, the cusp profile's dependence on species as well as the transmission efficiency of the cusp is investigated.

\section{B. Goals of preliminary study}

The work presented here are discharge data and preliminary cusp width measurements made on an arbitrarily selected magnet ring in a ring cusp discharge chamber. The discharge was operated at very low powers to carefully resolve both primary electron, plasma electron and ion contributions to plasma current line shape across a magnetic cusp at different heights above the anode to obtain a coarse map of the plasma collecting cusp. Azimuthal symmetry is assumed. The widths will be compared to a somewhat higher power case to look at the influence of space charge on collection width. At very low power, it is expected that the plasma itself may deviate from quasineutrality, as there are two sources of electrons: filament-derived primary electrons and secondary plasma electrons. In this case collection is expected to be dominated by negative space charge. At the higher powers, the ionization rate increases so ion production at the cusps can provide increased positive charge thereby modifying the collection width.

The measurement of the variation in loss width as a function of distance above magnets should yield insight into actual collection area. These species dependent measurements made by varying probe bias voltage are also presented. Finally, the width measurements made closest to the anode will be extrapolated and compared with measured burn line patterns and calculated widths as well. Follow on work will entail more detailed mapping as well as the incorporation of the inferred loss width based on spatial variation in the full width half maximum of current profiles in an energy and particle balance model such as that proposed by Lieberman ${ }^{10}$ and modified for ion thrusters by Goebel ${ }^{17}$. Burn line patterns observed on magnets will also be commented upon as they suggest differential heat loading and may by symptomatic of the various species and their average energies incident on the magnet.

\section{Experiment Design}

The design of the discharge chamber was dictated by the requirements of the magnetic circuit. A magnetic circuit desired that would use permanent magnet rings of alternating polarity such that the magnetic field on the anode surface would not drop below $30 \mathrm{G}$. In addition, the axial field magnitude was required to be a monotonically decreasing function of distance beyond the discharge chamber filament cathode. Such a gradient is associated with discharge stability. The magnetic circuit designed used four internally mounted samarium-cobalt magnet rings affixed to a truncated cone discharge chamber body constructed of mild steel. The magnetic field magnitude contour plot is given in Figure 1. Red lines indicated magnetic field amplitudes of $3 \mathrm{kG}$, and blue lines indicate magnetic field amplitudes of 30 G. The chamber is $20 \mathrm{~cm}$ in diameter at its exit plane and $8 \mathrm{~cm}$ in diameter at the upstream portion of the truncated cone. The fourth magnet ring is mounted on a two-segment flange on the exit plane of the chamber. The discharge chamber body and the magnet rings form the anode of the thruster.

A tungsten filament, fashioned from $0.2 \mathrm{~mm}$

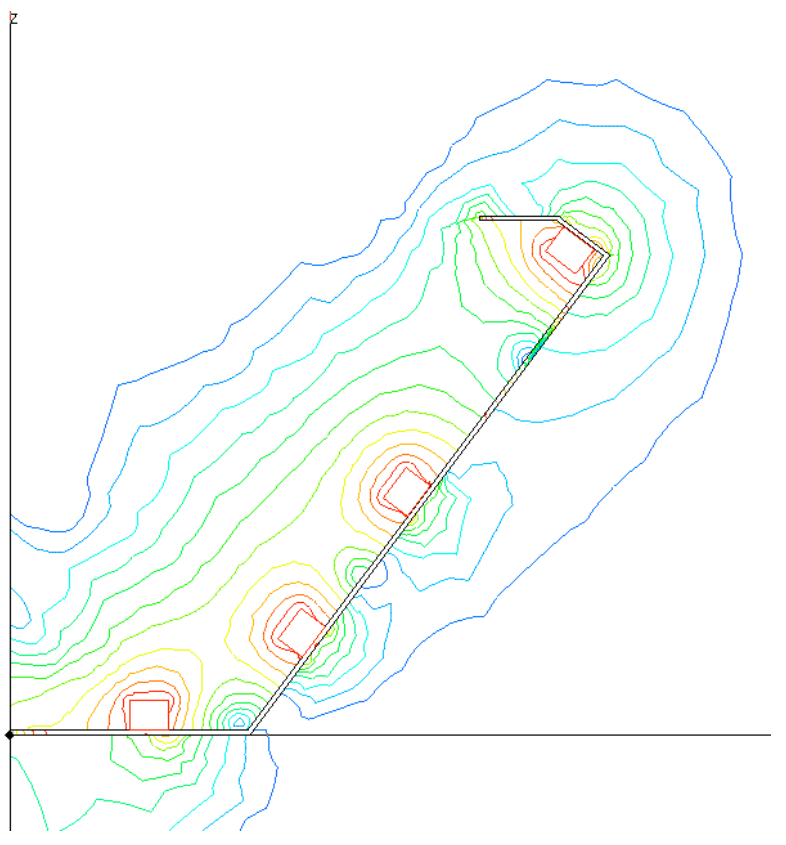

Figure 1. Magnetic field contour plot. tungsten wire, was used as the discharge chamber cathode. The filament, which was coated in R500 coating to improve thermal electron emission, was electrically isolated from the anode using ceramic insulators, as shown in Figure 2. Finally, a stainless steel mesh grid was mounted at the exit plane of the discharge chamber to collect ion 
current. The grid is mounted using ceramic standoffs to isolate it from the thruster anode. The discharge chamber and grid, shown in Figure 3, are supported by an aluminum mounting plate, and the entire setup is placed inside a vacuum chamber. Neutral argon gas is fed into the chamber through a gas plenum ring which is located inside the flange on the exit plane flange for reverse gas feed.

Three DC power supplies were used to power the source. One power supply was used to supply current to the filament. The discharge power supply was used to bias the anode at a positive voltage relative to the filament. The grid power supply was used to supply the grid with a negative voltage to determine ion saturation current at the exit plane of the source. The electrical connections were sent through an electrical feedthrough on the vacuum chamber, as illustrated in the wiring diagram shown in Figure 4.

Two probes were used to make measurements inside the discharge chamber. A fixed Langmuir probe was positioned near the center of the chamber to take bulk plasma measurements using an automated Langmuir probe. A second, translatable planar probe was used to measure plasma properties across one of the magnet rings, pictured in Figure 5. The cusp probe has a $1 \mathrm{~mm}^{2}$ tantalum collection surface. The probe was mounted on a Macor extension arm, which is fed through a hole in the grid and attached to a linear motion feedthrough. The feedthrough has a motion range of $25 \mathrm{~mm}$, and a precision of $0.01 \mathrm{~mm}$, allowing for closely spaced measurements. The "L" shape of the probe is intended to prevent shadowing from the probe body, which might otherwise block plasma from reaching the cusp. The cusp probe was moved across the third magnet ring, and could be adjusted to move across the cusp at a range of heights above the magnet surface. Cusp probe voltage was provided by a bipolar DC power supply. An electrometer was used to measure the probe current. Cusp probe currents were extremely small, ranging from $10^{-9}$ to $10^{-5} \mathrm{~A}$. A diagnostics wiring diagram is given in Figure 6.

The linear motion feedthrough was used to move the cusp probe in measured increments across the third magnet ring. In each probe sweep, the probe was moved a total distance of $13 \mathrm{~mm}$, a distance that was observed to capture the cusp spike in current, as well as the return to baseline on either side of the cusp. Current was measured at increments of no more than $0.1 \mathrm{~mm}$.
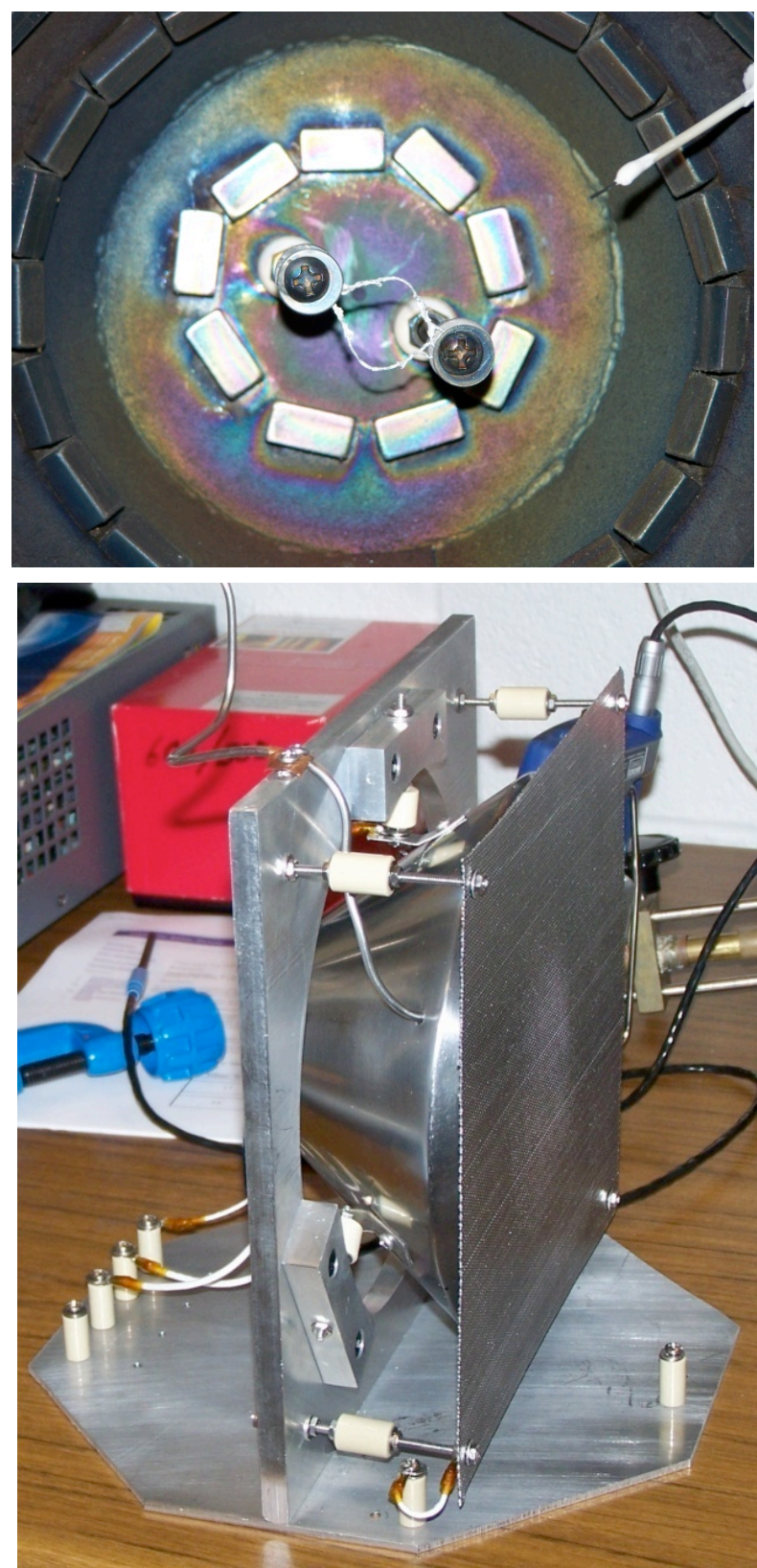

Figure 3. Discharge chamber with grid and aluminum mounting plate. 


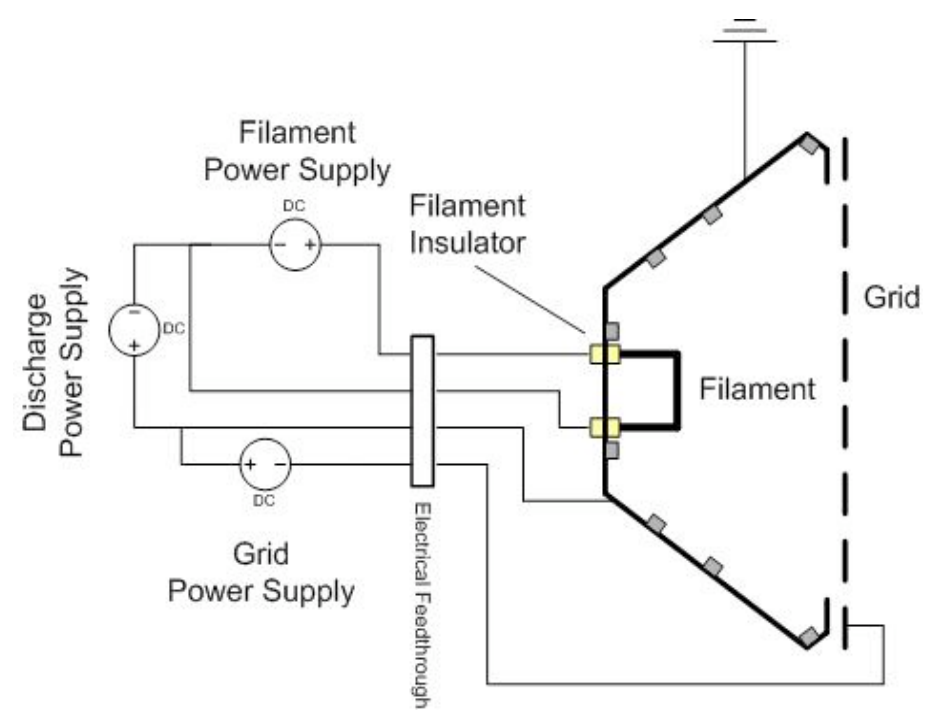

Figure 4. Discharge chamber wiring diagram.

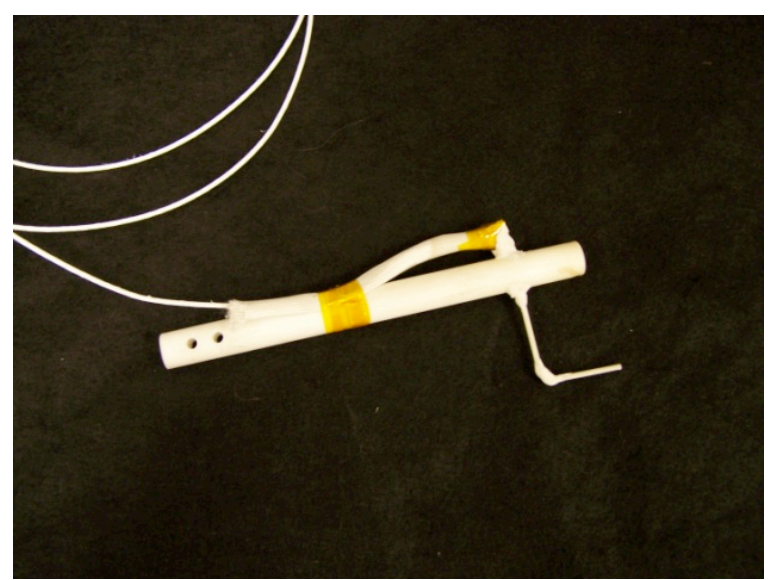

Figure 5. Cusp probe.

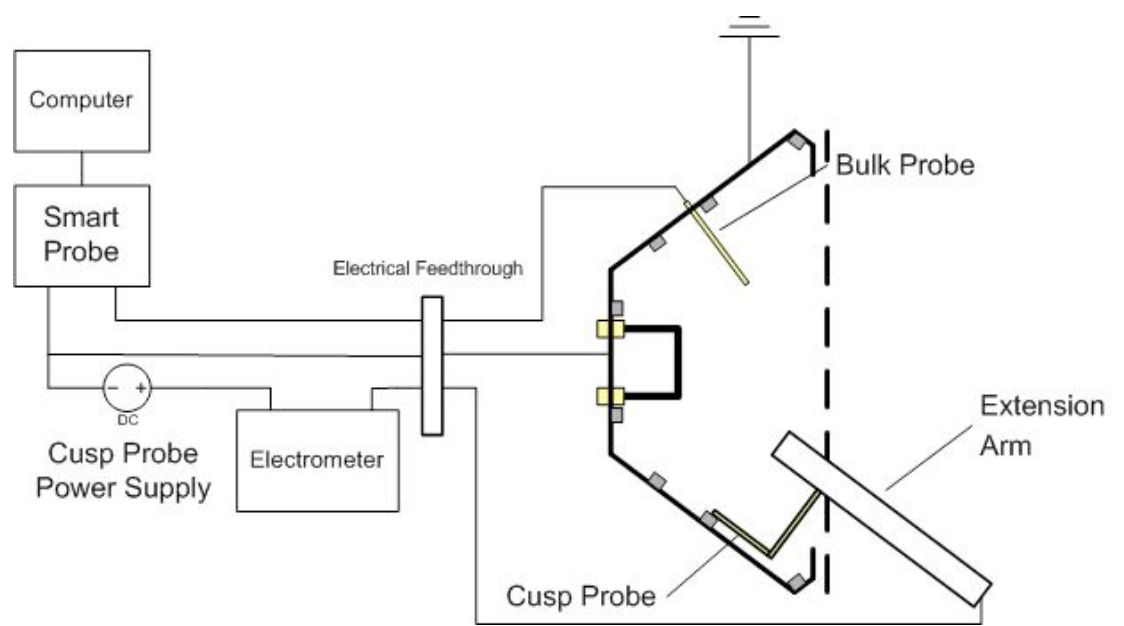

Figure 6. Probe wiring diagram. 


\section{A. Burn Lines}

During periods of extended operation, centered dark lines and at times colored bands appear on the surface of the magnets. These discolorations are associated with current collection. These patterns are referred to as "burn lines" in this work. Presumably, the burn line impressions are due to localized heat damage or electron induced oxidation at the surface of the magnet. These impressions no doubt represent the collection imprint in some fashion and are therefore are related to the plasma loss width at the anode.

Burn lines were observed on the surface of the magnet rings after running the source for several hours. An example of these burn lines is given in Figure 7. In an attempt to quantify these burn lines, the thruster was operated at controlled conditions and burn line width measured post test. The discharge was operated at high power $(40 \mathrm{~W})$ and low power $(5 \mathrm{~W})$ conditions. After each test, four magnets were collected from each of the four magnet rings $(90$ degree separation). Each magnet was imaged using a microscope and its burn line width measured. Averaging the burn line width across the four magnets gave an average burn line width for each magnet ring at each of the two conditions.

The analysis of burn line widths from the third magnet ring is discussed in detail later in this work. This ring was interrogated by the cusp probe. Consequently, the burn line width at this ring could be compared to actual current density profiles across the magnetic cusp. At high power, the average burn line width at this ring was $0.525 \mathrm{~mm}$. At low power, the average burn line width was $0.388 \mathrm{~mm}$.

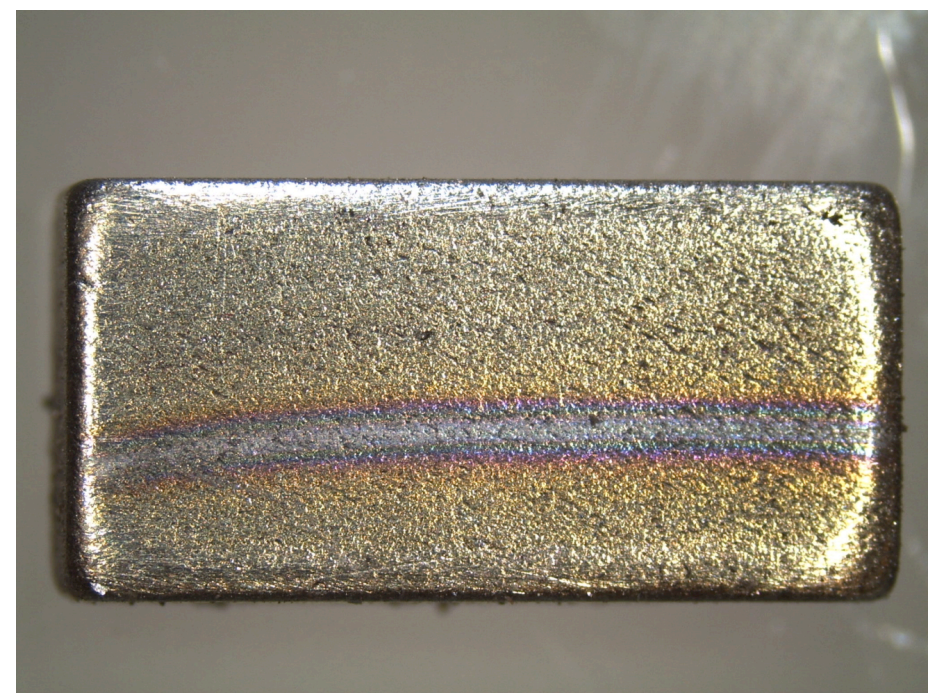

Figure 7. Image of a magnet after running the discharge chamber for several hours.

\section{Data Analysis}

Discharge characteristics of the 4-ring, filament-driven ion source were obtained as a function of discharge power and pressure. The discharge was typically operated in constant voltage mode. For these experiments, it was desirable to operate with the discharge current fixed as well. The discharge current was made constant by adjusting the filament current. The discharge ion production efficiency could be assessed by measuring the ion current collected at the grid as a function of the discharge current. Figures $8 \mathrm{a}$ and $8 \mathrm{~b}$ illustrate the discharge behavior and ion production at two different argon flow rates.

At fixed discharge voltage, as expected with increasing filament current, the discharge current and thus the ion production rate monotonically increase as inferred from the increase in collected grid ion current. The rate of increase is considerably more rapid for the higher flow rate case at increased discharge currents, owing to the linear dependence of the ionization rate on both the neutral density and the primary electron density. 

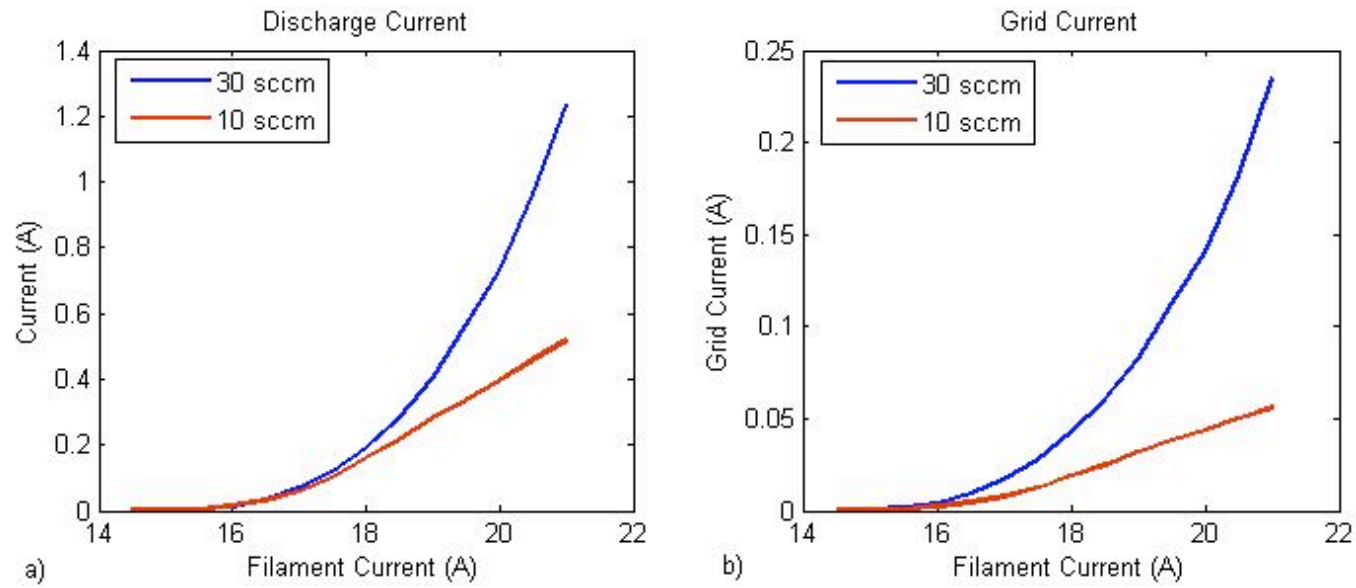

Figure 8a. Discharge current as a function of filament current at two different flow rates. Figure 8b. Grid current as a function of filament current at two different flow rates.

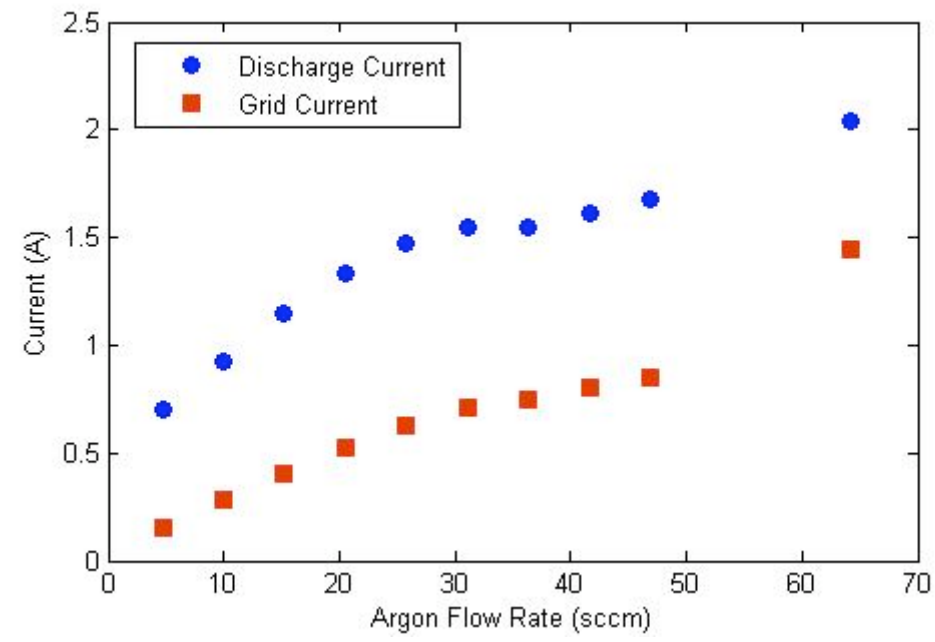

Figure 9. Discharge and grid current as a function of argon flow rate.
Figure 9 illustrates variations in the discharge current and ion current with flow rate at a fixed discharge voltage of $60 \mathrm{~V}$. Ion currents as high as nearly 1.5 A were collected at very high flow rates. The trend in collected ion current follows that of the discharge current as expected. One can calculate the discharge ion production cost from this data as a function of flow rate. Of the operating points investigated here, the ion production costs ranged from as low as $133 \mathrm{~W} / \mathrm{A}$ to $250 \mathrm{~W} / \mathrm{A}$. These numbers are surprisingly good for this small source. For these initial tests, no attempt was made to optimize discharge performance. Over all conditions investigated, the discharge was stable.

Future efforts will involve further assessing the discharge chamber by correcting for ingested flow, the utilization of xenon rather than argon, and the use of a hollow cathode rather than a filament. These changes should improve overall discharge performance. As a test bed for cusp studies, however, the use of a filament discharge is somewhat ideal in that at low gas utilization (typically $<0.5$ ), the convecting current is mostly primary electrons. It is therefore possible to assess collection widths in the limit of no gas flow. This cannot be done with a hollow cathode due to the high collisionality in the orifice.

A set of standard operating conditions were established. These operating conditions were held constant throughout the experiments discussed herein by adjusting the filament current. Discharge voltage was set to $50 \mathrm{~V}$. The filament current was controlled such that a discharge current of about $0.1 \mathrm{~A}$ was maintained, resulting in a discharge power of about $5 \mathrm{~W}$. The grid was held at a bias of $-30 \mathrm{~V}$. Langmuir probe analysis indicated a bulk plasma electron temperature of around $13.4 \mathrm{eV}$ and densities on the order of $1 * 10^{9} \mathrm{H} / \mathrm{cm}^{3}$. Again, this low utilization condition was used to contrast differences between plasma electron and primary electron collection at the anode. Higher power conditions were also investigated for comparison purposes.

\section{A. Bulk Plasma Properties}

Bulk plasma properties were assessed using the Langmuir probe that was mounted such that it probed plasma properties in the middle of the discharge chamber. Langmuir probe data was taken at each discharge condition at which cusp data was acquired. The magnetic field at the probe location was approximately $50 \mathrm{G}$. The electron 
temperatures investigated in this work indicate electron Larmor radii on the order of $5 \mathrm{~mm}$, thereby satisfying condition that the Larmor radius is large compared to the dimensions of the probe. Trends in plasma conditions with changes to the discharge made by the operator are expected to be well represented by the probe.

Figure 10 illustrates Langmuir probe data acquired at the operating conditions for which the majority of the cusp data was acquired. Here the discharge current was approximately $0.1 \mathrm{~A}$, the discharge voltage held at $50 \mathrm{~V}$, the flow rate was $30 \mathrm{sccm}$ and the vacuum chamber pressure was about 5 mTorr. The ion saturation current was subtracted away from every point on the curve to yield the electron current. Quite noticeable from this trace is the rather extended linear region extending from roughly $-50 \mathrm{~V}$ to nearly $-5 \mathrm{~V}$. This extended region is due to the presence of primary electrons ${ }^{18}$. Extrapolating from the zero electron current point to plasma potential yields the potential difference associated with the primary electron energy. This difference as expected is approximately equal to the discharge voltage.

The pronounced primary electron presence in the IV characteristic is not surprising. In contrast to hollow cathode arc discharge, in filament discharges the primary electrons do not encounter collisions during extraction. In a hollow cathode, the collisional sheath damps out the amplitude of the primary electron signal. The majority of the electrons emanating from the hollow cathode are produced in the orifice due to primary electron collisions with neutrals there. In the filament case the electrons are injected directly into the discharge at full energy. The presence of the primary electrons can distort the overall IV characteristic yielding uncertainty in the electron temperature measurement. To get a rough idea of scaling, in this work the linear region of the low temperature portion is used

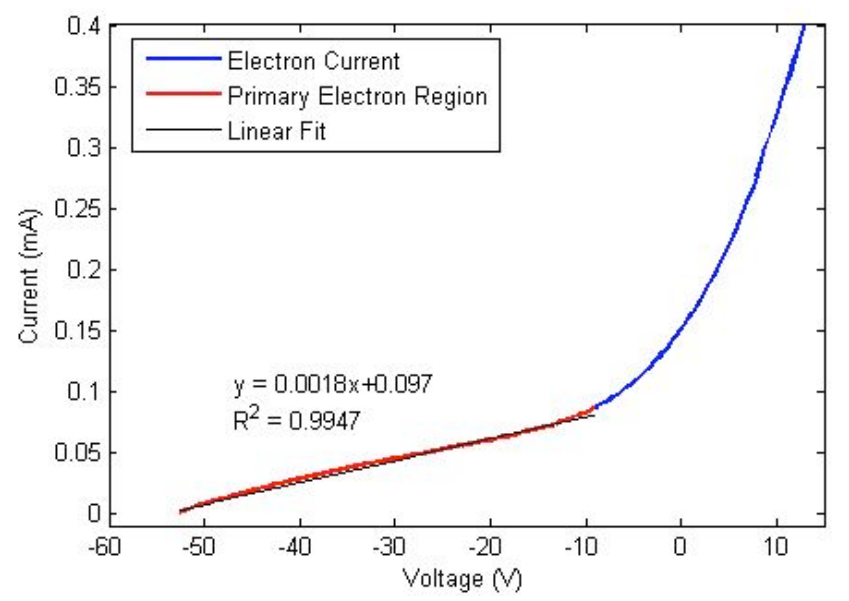

Figure 10. Bulk Langmuir data at nominal operating conditions.

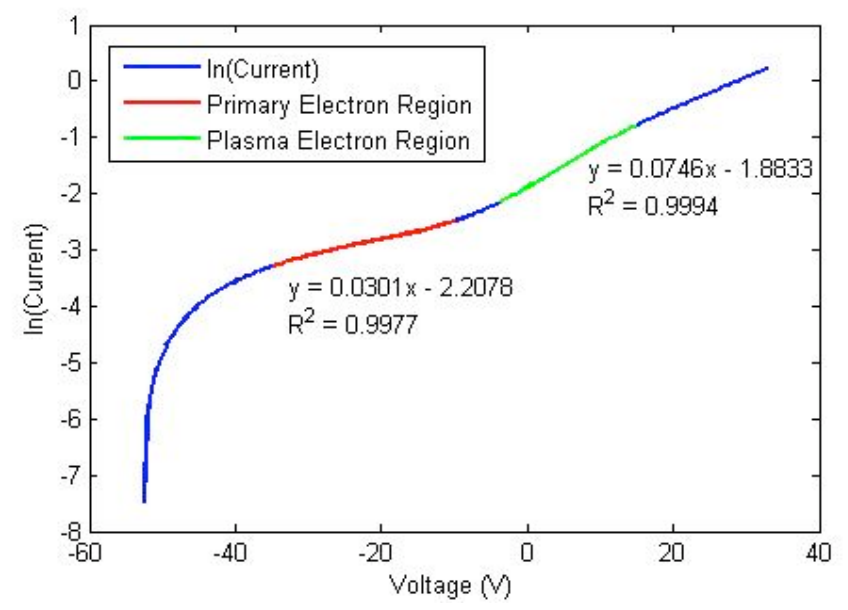

Figure 11. Natural logarithm of bulk Langmuir data at nominal operating conditions.

to assess the plasma electron temperature. No attempt was made to subtract out the primary electron contribution as it is known that this approach is subject to error ${ }^{18}$. Future investigations will entail a more detailed analysis of the electron energy distribution function. Figure 11 illustrates a plot of the natural logarithm of the same data presented in Figure 10 as a function of voltage. From this data it is readily observable that there are two distinct linear regions, with the slope of each region increasing with voltage. The first region (smaller slope) represents the energetic primary electrons. The second region corresponds to the bulk electrons. The slope of the second region yields a plasma electron temperature of $13.4 \mathrm{eV}$. This temperature is consistent with similar filament discharge experiments ${ }^{19}$. The subtle knee just beyond the bulk electron region represents another region but of reduced slope. This region corresponds to the expanding electron saturation region. The transition knee between these two regions represents the plasma potential, which apparently occurs near $15 \mathrm{~V}$. In this work, the cathode fall voltage was taken to be of the order of the discharge voltage $(50 \mathrm{~V})$; consequently, the energy of primary electrons is taken as $50 \mathrm{eV}$. Primary electrons were evident in each probe measurement taken at the conditions investigated in this work.

In order to assess electron collection widths due essentially to the primary electrons, the discharge was also operated without any gas flow. Langmuir probe traces using the bulk plasma probe were acquired for this condition as well. In this case, very few ions were expected to be produced. This is consistent with observed Langmuir probe $\mathrm{I}-\mathrm{V}$ data, an illustrative example of which is shown in Figure 12. No evidence of a well defined lower temperature 
population was found from the analysis of this trace. The noisy, relatively flat region beyond $-30 \mathrm{~V}$ is taken to be equal to zero. The trace, though noisy, has the appearance of an extended primary electron region. The voltage difference between the zero of the curve and plasma potential yields the energy of the primaries. In this case however the plasma potential knee is not well defined. Rather, if one assumes that the primary electron energy is approximately equal to the discharge voltage (times e), then one can add 50 volts to the zero crossing point to ascertain the plasma potential, which yields about $20 \mathrm{~V}$ in this case.

\section{B. Cusp Data Discussion}

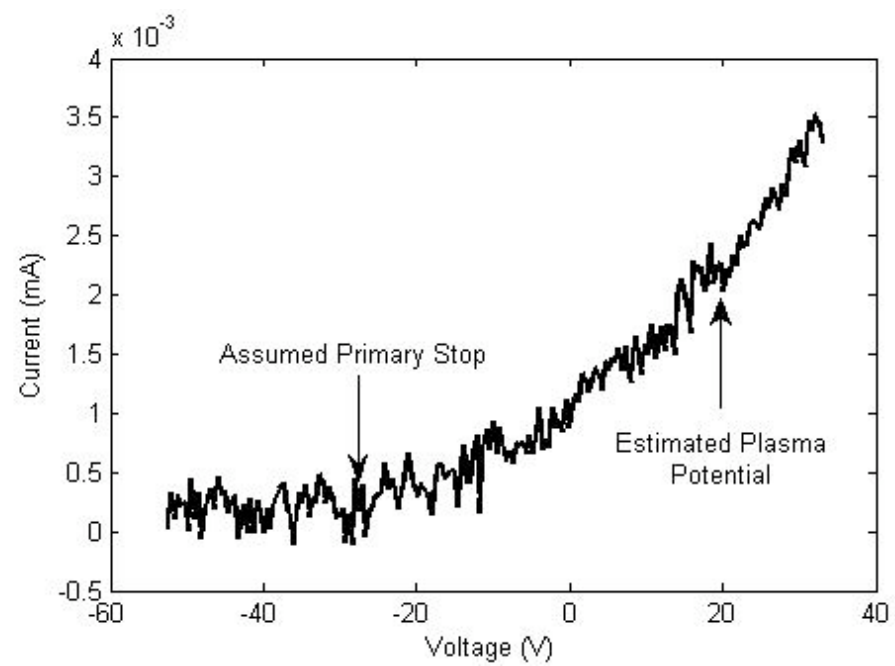

Figure 12. Bulk Langmuir data when no plasma is present.

One major goal of this ongoing effort is to ascertain the relationship between cusp collection structure and discharge efficiency. As mentioned earlier, the magnetic cusp itself may be casually thought of as a collection funnel with the mouth opening into the field-free region of the bulk plasma. Plasma particles enter this collection mouth and are ultimately transported to the wall. The funnel itself has a loss cone associated with it. Some particles entering this portion of phase space are transported to the wall, while others are simply reflected. In this regard, the collection funnel has associated with it a velocity dependent transmission coefficient that is also dependent on the spatial distribution of the magnetic cusp. The collection loss cone can be thought of as the protrusion of the bulk plasma to the wall, shaped by the magnetic cusp. In this regard, the cusp region is actually a sheath structure as it is a transition vehicle between the bulk plasma and the wall. The preliminary work presented here maps out the spatial distribution of charged particles along a magnetic cusp axis at different heights above the magnet ring. Presented here are results of a preliminary coarse survey. The spatial distribution of plasma current across a given magnetic ring is a sampling of the density distribution at that particular height. These spatial distributions are called collection widths and in general can vary in shape depending on species. In this work, shape is found to also vary as a function of height above the magnet ring. The scanning probe used in this work was biased at different voltages in an attempt to distinguish between the collection widths of the various species. In this work, the effort focused on examining differences between primary electron, plasma electron, and ion collection widths. In most cases, the widths were a combination of species. Also investigated were conditions without gas flow to examine primary electron collection widths only. To achieve aforementioned objectives of this study, a special effort was made to operate the discharge chamber at low powers $(5 \mathrm{~W})$ to achieve sharper contrast between plasma and primary electrons. For comparison, a high power case was also investigated (40W).

\section{Selecting Bias Voltages for Cusp Probe.}

In order to determine the bias voltage to apply to the scanning probe surface, a Langmuir probe trace was taken at a height of $1 \mathrm{~mm}$ above the surface of the magnet ring. This trace was used to determine roughly where the electron and ion saturation currents occurred. Figure 13 illustrates the variation in the collected current as a function of bias voltage for a typical operating condition investigated.

As can be seen in the figure, the ion current appears to saturate at voltages greater than -20 volts. A bias voltage of $-30 \mathrm{~V}$ was applied to the probe to assure repulsion of most of the plasma electrons. The bias voltage of $-30 \mathrm{~V}$ is over two times the estimated energy of the plasma electrons. At $-30 \mathrm{~V}$, it is therefore expected that the collection width should be only a mixture of primary electrons and plasma ions. Further inspection of the I-V trace indicates the knee of the curve occurs in the vicinity of $5 \mathrm{~V}$, though there is significant rounding. Here at the probe location, the plasma potential is approximately 5 Volts above anode potential. Therefore, probe bias voltages above $5 \mathrm{~V}$ should be sufficiently high to repel ions. In this regard, the loss width of the combination of the primary and plasma electrons only should be attainable at bias voltages greater than $5 \mathrm{~V}$. Ten volts was used to achieve this. 


\section{Cusp width measurements}

In general, the current profiles obtained when the probe was swept across a magnet element making up the magnet ring turned out to be surprisingly Gaussian. This is consistent with measurements conducted in the past ${ }^{1}$. In all cases, the current profiles tended to narrow as the anode was approached, indicating increased peak current density. The narrowing is associated with the physically narrow structure of the magnetic cusp at the anode surface. It should also be pointed out that the sweep across an individual magnet element making up the ring is expected to be representative of profiles across all magnets that make up that particular ring, as one would expect from symmetry. The majority of the data was taken at the $30 \mathrm{sccm}, 5 \mathrm{~W}$ operating condition. For comparison profiles were also obtained without gas flow and at considerably higher power $(40 \mathrm{~W})$.

\section{E. Nominal Case: 5 W, 30 SCCM}

The current profiles at the $5 \mathrm{~W}$ case are shown in Figures 14a-d and 15a-d. Data acquired at $-30 \mathrm{~V}$ probe bias is shown in Figure 14 at four different heights above the magnet cusp, and data acquired at $10 \mathrm{~V}$ is shown in Figure 15 at the same four heights. As can be seen here, peaks are reasonably symmetric, though not quite located over the magnet centerline with the exception of the $10 \mathrm{~mm}$ data. One possible explanation for this is asymmetries in the
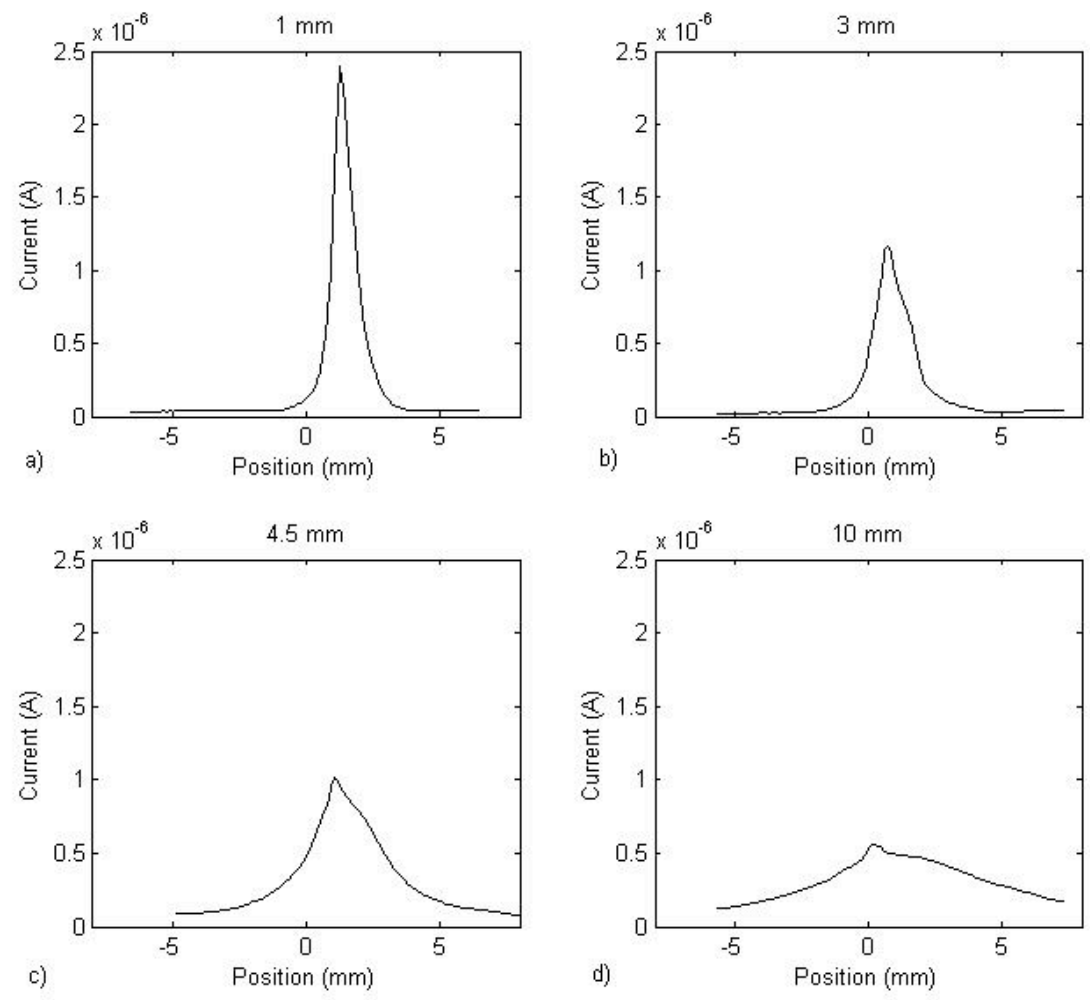

Figure 14a-d. Cusp current profiles at $-30 \mathrm{~V}$ at four different heights above the magnet surface.

American Institute of Aeronautics and Astronautics 092407 

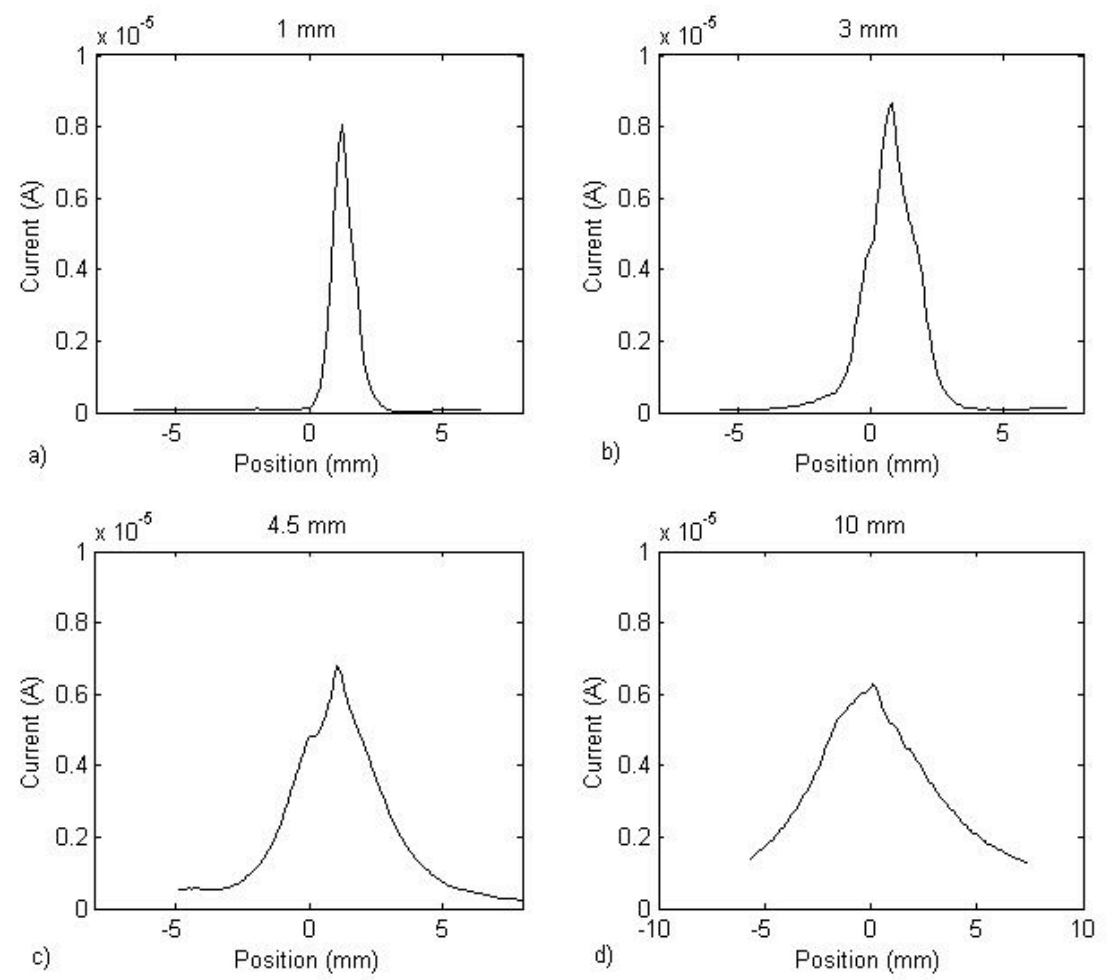

Figure 15a-d. Cusp current profiles at $10 \mathrm{~V}$ at four different heights above the magnet surface.

magnetic field over the cusps that presumably increase at distances closer to the magnet surface. The peak widths broaden with increasing height above the magnetic cusp. A flat baseline region is visible on each side of the peaks, except for the data taken at a probe height of $10 \mathrm{~mm}$. The lack of a baseline at this height is likely due to the probe track being too short to capture the entire distribution. Future work will correct for this by using a linear translation stage with a longer travel. The peaks appear Gaussian, though the top of the peak is sharper than what would be expected from a Gaussian distribution. This is attributed to the spatial distribution of the field lines that make up the magnetic cusp. The widening of the profile with increasing height is consistent with expected behavior of the melding of the collection cusp with the bulk plasma. Extrapolation of the cusp shape into the bulk plasma suggests again the funnel like shape but rotated azimuthally to reveal the actual profile. When the field at the mouth of the profile reaches the value of the nearly field-free bulk region or plasma boundary, one has effectively reached the mouth of the cusp. The four heights and corresponding average magnetic field strength at those heights are: $3 \mathrm{kG}$ at the magnet surface, $2125 \mathrm{G}$ at $1 \mathrm{~mm}$ above the magnet surface, $1640 \mathrm{G}$ at $3 \mathrm{~mm}, 1060 \mathrm{G}$ at $4.5 \mathrm{~mm}$, and $620 \mathrm{G}$ at 10 $\mathrm{mm}$.

Figure 16 presents a calculated contour plot of the magnetic field around the magnetic cusp investigated. Red lines indicate a magnetic field strength of $3000 \mathrm{G}$, and blue lines indicate a magnetic field strength of $50 \mathrm{G}$. As can be seen from the figure, if one assumes for example that the 100 Gauss line is the boundary of the discharge, then the mouth of the magnetic cusp is located at about 20 to $30 \mathrm{~mm}$ above the magnet surface. Future investigations will involve more detailed characterizations of this region both using translating plasma probes and a Hall probe to obtain better insight into this region. One goal is to use this detailed data to calculate the loss cone of the cusp for incident particles diffusing from the bulk plasma.

The $-30 \mathrm{~V}$ bias should be sufficiently high to reject most of the plasma electrons, leaving only the ion and primary electron current. The loss width closest to the wall is expected to reflect the width of the "funnel" drain into the anode. The Gaussian-like current profiles allowed for relatively straightforward determination of the loss widths. These were taken to be the full width at half maximum (FWHM) of the profiles. Figure 17 shows the variation in the width as a function of height. $10 \mathrm{~mm}$ data was omitted. This was done because the profile at this height was so broad making it difficult to assess the FWHM over the sweep range used. Future positioning apparatus will allow for larger travel. 


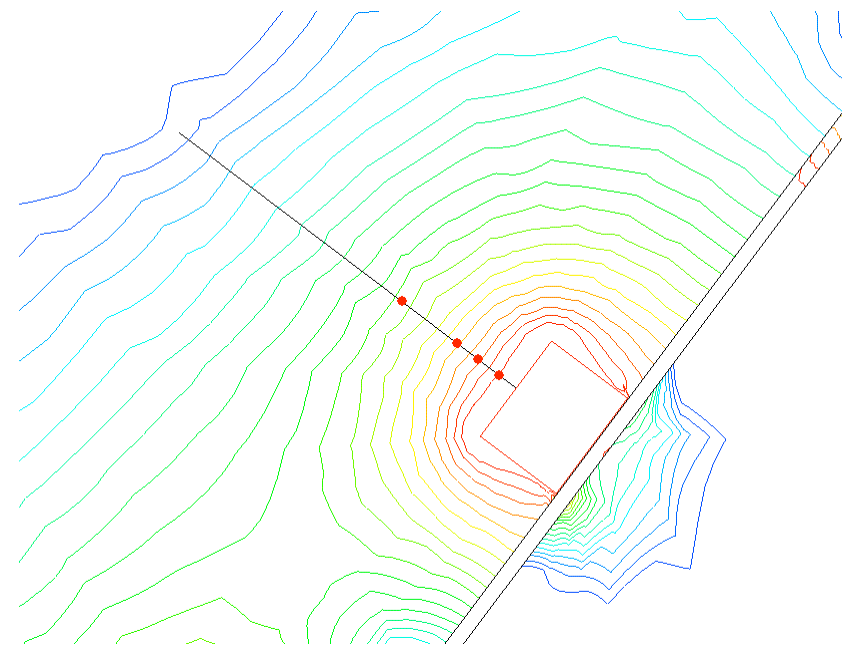

Figure 16. Contour plot showing magnetic field strength above third magnet ring (log scale). Red dots indicate probe heights in cusp data $(1,3,4.5$, and $10 \mathrm{~mm})$.

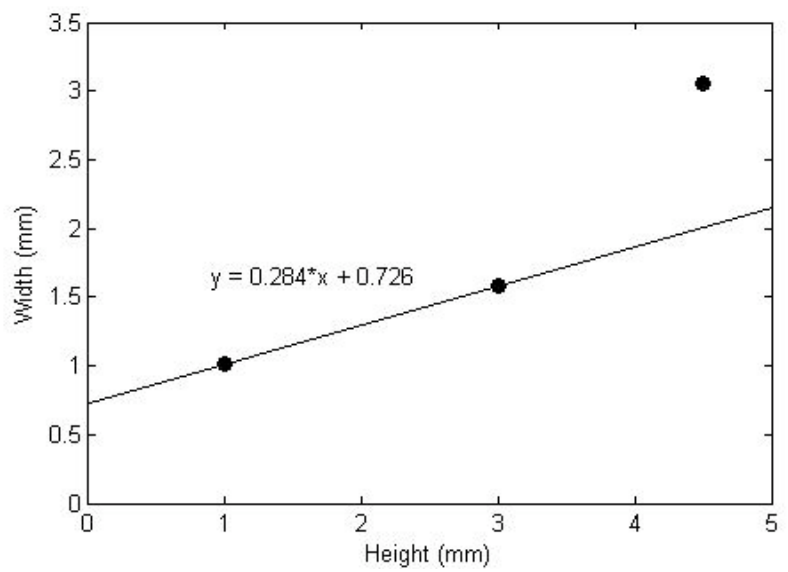

Figure 17. Peak width described by FWHM as a function of probe height above magnet surface for $-30 \mathrm{~V}$.
Extrapolation of this profile to the anode itself should yield the actual loss width at the anode. The relative coarseness in height data does not permit an accurate determination of the functional dependence of the loss width with height. In this work, to obtain an estimate of the loss width at the surface of the magnet, the two data points taken closest to the anode surface are used to extrapolate the value to a height of zero. This rather crude estimate is expected to yield an upper limit on the width since the magnetic field increases exponentially rather than linearly with decreasing distance from the magnet surface ${ }^{2}$. In the case of the $-30 \mathrm{~V}$ bias, a linear fit using these two points yields a width of approximately 0.7 $\mathrm{mm}$, quite close to the hybrid radius. Based on these measurements, the collection width apparently still depends on plasma electrons through the hybrid radius despite the fact that the probe was biased such that these electrons are prevented from collection at $-30 \mathrm{~V}$. This should be of no surprise since the collection width profile is still determined by electrostatic interactions between plasma electrons, primary electrons, and ions in the cusp as discussed by Knorr and Merlino ${ }^{20}$. Self-consistent fields set up between ion and electron flows can allows for broadening of the electron footprint and narrowing of the ion footprint. This is true regardless of the probe bias so long as it is sufficiently non-perturbative. This means that the measured widths should be relatively insensitive to bias voltage. The probe merely samples this self-consistent profile at each height level. The biased probe, however is sensitive to specific species and this is reflected through measured current. Changes in peak height give much insight into species propagation efficiency through the magnetic cusp.

Of great interest is just how the current varies through the collecting cusp as a function of height in the cusp. In general, it is not obvious that all the particles that enter the cusp actually end up at the anode. After all, associated with the cusp is a collection loss cone. This collection loss cone has significant implications on efficiency as it predominantly determines the true losses to the wall, and not the loss width measured at the wall. The measurements made in this work yield some insight into the collection efficiency of the magnetic cusp. Future work will investigate the loss cone itself. By integrating the area under the collection profiles obtained from sweeps across the magnetic cusp, one obtains a parameter that is proportional to the current flowing across that particular investigation plane. These integrations were obtained to determine how much of the plasma that enters the mouth of the collection cusp actually ends up at the anode. Examination of the $-30 \mathrm{~V}$ data yields much insight into this current flow of primaries and plasma ions. Based on the comparison between the integrated current calculated at $10 \mathrm{~mm}$ above the cusp to that calculated at only $1 \mathrm{~mm}$ above the cusp, about $80 \%$ of the current actually makes it to the anode wall. This finding suggests modest attenuation of plasma current as it propagates through the cusp. Again, this is postulated to be due to the loss cone and the particle's position in phase space. Electrons that do not make it to the surface are apparently reflected by the mirror action of the cusp. Again, this data indicates that clearly it is not the area at the wall that determines the losses and thus efficiency. Rather, it is the collection and transport efficiency of the magnetic cusp. It is interesting to note that the mirror properties of the cusp can be calculated somewhat 
independently of the plasma to get a rough understanding of transport losses through the cusp. Such a calculation is a goal of this ongoing work.

Integrated areas and loss widths were also determined for the +10 Volt data, shown in Figure 18 and Figure 19. As can be seen in Figure 18, the current drop off is linear close to the magnet surface. Again this data indicates current loss as the plasma flows from the discharge to the anode wall. This data evokes the concept of the cusp as a velocity selective filter, allowing only those with the correct parallel and perpendicular velocity components to actually enter the anode. The particles are spatially separated according to the velocity vector at that given magnetic topology just before impact at the magnet. From this data, it can be seen that for the $+10 \mathrm{~V}$ bias case the current decreases as one approaches the wall by nearly $80 \%$, indicating that plasma current is significantly attenuated as it propagates through the magnetic cusp on the way to the anode wall. This attenuation is much greater than that observed in the $-30 \mathrm{~V}$ case. The only difference between the two cases is the presence of the plasma electrons and absence of the ion current in the $+10 \mathrm{~V}$ case. When the probe was biased above plasma potential, the collected current was an order of magnitude higher than the case where the probe was biased at $-30 \mathrm{~V}$. A possible explanation for the discrepancy in the transmission efficiencies at the different bias voltages is related to the presence of the plasma electrons. While a great deal of these electrons enter the cusp, a significant fraction of them lack the sufficient parallel velocity to actually reach the anode. The plasma electron current contribution therefore experiences severe attenuation along the way to the anode. The primary electrons, on the other hand, are perhaps more capable of reaching the anode as they can be expected to possess high perpendicular and parallel velocity components. The transmission efficiency in this context is therefore meaningful so as long as one refers to a specific species. In this case, the smaller transmission fraction is presumably due to the significant drop-off in plasma electron current as the current propagates to the anode. Likely, the transmission efficiency of the primaries remains at about $80 \%$ in accordance with the $-30 \mathrm{~V}$ data. Future measurements will be carried out to verify these effective transmission coefficients. It may be possible to decrease the

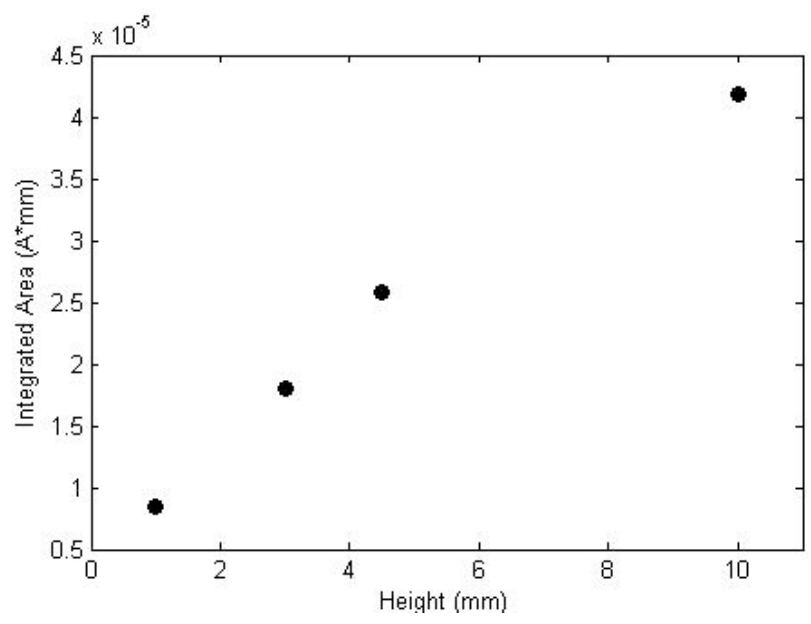

Figure 18. Integrated area as a function of probe height at $10 \mathrm{~V}$.

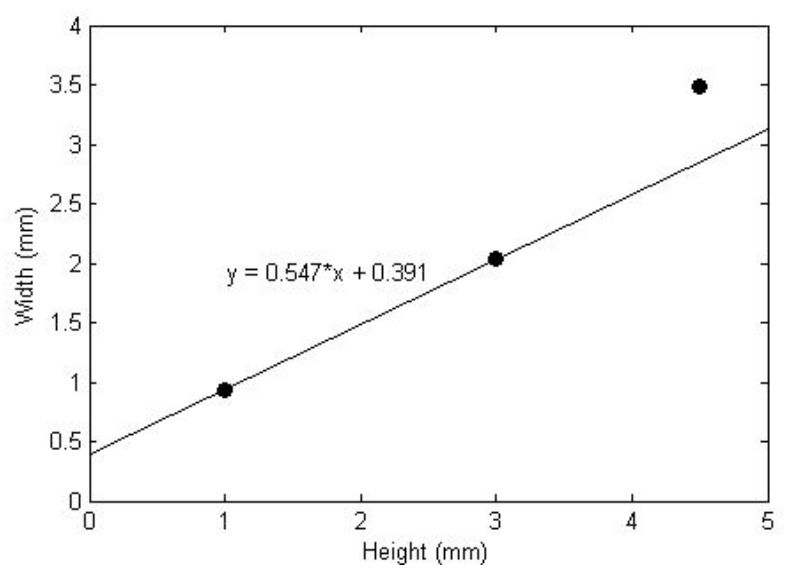

Figure 19. Peak width described by FWHM as a function of probe height above magnet surface at $10 \mathrm{~V}$.

primary electron transmission coefficient further by simple modifications to the magnetic cusp. By balancing such reductions with discharge stability, one should be able to improve performance by relatively straightforward magnetic circuit modifications.

For the $10 \mathrm{~V}$ bias data, we expect to see only primary and plasma electrons collected. At $10 \mathrm{~V}$, the ions should be repelled based on "at-cusp" Langmuir probe measurements. Figure 19 illustrates the variation in the loss width with distance. Extrapolation to a height of zero again using the two closest points indicates a slightly narrower cusp loss width of approximately $0.4 \mathrm{~mm}$, which is roughly 60 percent of that which was measured when the probe was biased negatively. The relative closeness of this value with the negatively biased probe, taking into account the relative crudeness of the extrapolation, again suggests that the bias of the probe does not appreciably affect the profile as the profile is determined strictly by the flow of plasma particles self-consistently through the cusp. The extrapolated value also agrees within $5 \%$ of the magnet burn line width. In this case the extrapolated width is somewhat smaller than the hybrid radius. Future work will involve using sweeps at a larger number of heights to 
better capture the functional loss width, particularly near the magnet surface. Such data will yield a higher resolution snapshot of the magnetic cusp. This should lead to better extrapolation to the anode wall. In any case, the measurements suggest loss widths at the anode correspond most closely to the hybrid radius.

It is also worth comparing the measured FWHMs obtained at the $-30 \mathrm{~V}$ and $+10 \mathrm{~V}$ at the various heights above the anode magnet that were investigated. The collection widths at these heights can be compared with the hybrid radius. Hybrid radius was calculated using magnetic field values measured at different heights above the anode using a Hall probe. Electron temperature was taken to be either the plasma electron temperature of $13.4 \mathrm{eV}$, or the primary electron temperature of $50 \mathrm{eV}$. The ion Larmor radius was found using the Bohm velocity, $\mathrm{C}_{\mathrm{s}}$, as the perpendicular velocity component ${ }^{1}$, and the cyclotron frequency, $\omega_{\mathrm{ci}}$ :

$$
r_{\mathrm{i}}=\frac{c_{s}}{\omega_{c i}} \text { where } C_{s}=\sqrt{\frac{k T_{g}}{M_{i}}}
$$

Figure 20 depicts these comparisons for the $-30 \mathrm{~V}$ data and the $+10 \mathrm{~V}$ data. Error bars are calculated based on uncertainty in the magnetic field strength measurements. The data appears to be bracketed by the hybrid radius calculated using the primary electron energy $(50 \mathrm{eV})$, a rough upper limit, and the plasma electron energy (13.4 $\mathrm{eV}$ ), a lower limit. Burn line width at the anode is also plotted for comparison at a probe height of zero $\mathrm{mm}$. Agreement appears best closest to the anode surface. Though the magnitudes are approximately similar to the calculated hybrid widths, the data is displaced appreciably from this bracketed region at the highest height investigated. Some of the deviation may be attributed to uncertainty in the assumed magnetic field strength at this location. At such distances, the radial component is significant. A three axis probe can be expected to yield a more accurate measurement of the field at this location than using the single axis probe which was used in this investigation to estimate the field. $+10 \mathrm{~V}$ data exhibits the strongest deviation.

\section{F. High Power Condition}

For comparison, cusp width measurements were also taken at the same flow rate but higher discharge power. The discharge power was increased from $5 \mathrm{~W}$ to $40 \mathrm{~W}$, thereby increasing the ionization fraction. This was achieved by increasing the discharge current by nearly a factor of 7 and the discharge voltage by an increment of nearly $10 \mathrm{~V}$. Bulk electron temperature differed between the low and high power cases by only 10 percent, which was within the uncertainty of the measurement. In this respect, one would expect only small differences between the cases based on temperature and primary electron energy considerations alone. An increase in the associated ion current flowing through the cusp at the higher power conditions might play a larger role in determining the cusp width at a given height above the magnet ring. Increased ion space charge in the collection cusp can affect the radial electric fields there, thereby affecting the width $^{20}$. Data was taken at only one height $(1 \mathrm{~mm})$ but both at $-30 \mathrm{~V}$ and then at $+10 \mathrm{~V}$ probe bias. At $-30 \mathrm{~V}$, the loss width of the higher power case was 20 percent larger than the lower power case. At $10 \mathrm{~V}$, the loss width of the higher power case was approximately 12 percent higher than the low power case. These measurements indicate that ion current may be playing larger role in collection. More detailed studies of charge variation and radial electric field are necessary to paint a clearer picture of how width varies with increased current flow. Additionally, primary electron energy and plasma electron temperature must be similar to obtain a clean comparison. The burn line width at the anode also increased slightly from approximately $0.4 \mathrm{~mm}$ at the low power case to $0.53 \mathrm{~mm}$ at the high power case, an increase of just over 20 percent. Again, the changes are small. 


\section{G.Primary Electron Only Data}

To gain better insight into the character of current collection at the cusps, the flow rate was reduced to zero sccm. Under these conditions, electron collection at the anode is almost entirely due to primary electrons. In this limit, one would expect that the loss width and burn line widths should all agree with the primary electron Larmor radius as positive space charge should be absent. These operating conditions were acquired at very low powers around $20 \mathrm{~mW}$. The space charge limited current flow in the absence of a plasma kept discharge currents below 1 mA. Nonetheless, measurable current profiles acquired across the magnetic cusps were possible using the electrometer. Figures 21a-d illustrates the variations in the peak shape with height above the cusps for a bias voltage of $+10 \mathrm{~V}$. All peaks are considerably narrower than those measured with a plasma at the same height above the magnets. Additionally, the peaks have different structure than the sweeps obtained when plasma is present. The left hand side of these peaks suggests an asymmetrical profile. One plausible explanation for the presence of this extended shoulder may be found by simply looking at where this distorted region is detected during the spatial sweep. It occurs always on the side of the magnetic cusp facing the filament cathode. This suggests that primary electron density is apparently attenuated laterally (along the probe surface normal) across the cusp. The probe is merely detecting this profile. This may not be so surprising as it implies that electrons injected along field lines into the magnetic cusp region has a higher intensity on the side of the cusp facing the filament simply due to the fact that the incident electron flux is stronger there, particularly at higher heights. This gives rise to the exaggerated profile in which the shoulder extends toward the filament cathode as observed in the data. Again, this effect should be more pronounced at greater distances above the magnet ring surface. The data bears this out as shown in Figure 21 where at a probe height of $1 \mathrm{~mm}$, the shoulder is essentially absent in contrast to the profile obtained at a probe height of 10 $\mathrm{mm}$. The regions closer to the magnet or anode surface are essentially magnetically insulated from such direct injection effects. The exaggerated shoulder profile is likely present in all the data at the higher heights even when plasma is present but the signal magnitude is likely too small to be resolved. This data again contrasts primary electron injection into cusp with plasma particle injection into the cusps. Primary electron injection as suggested by this data is in part directional, while the plasma injection is not. Figure 22 illustrates this point, showing attenuation depicted schematically across the cusp. Slight asymmetries in the magnetic field of the magnetic cusp due to the
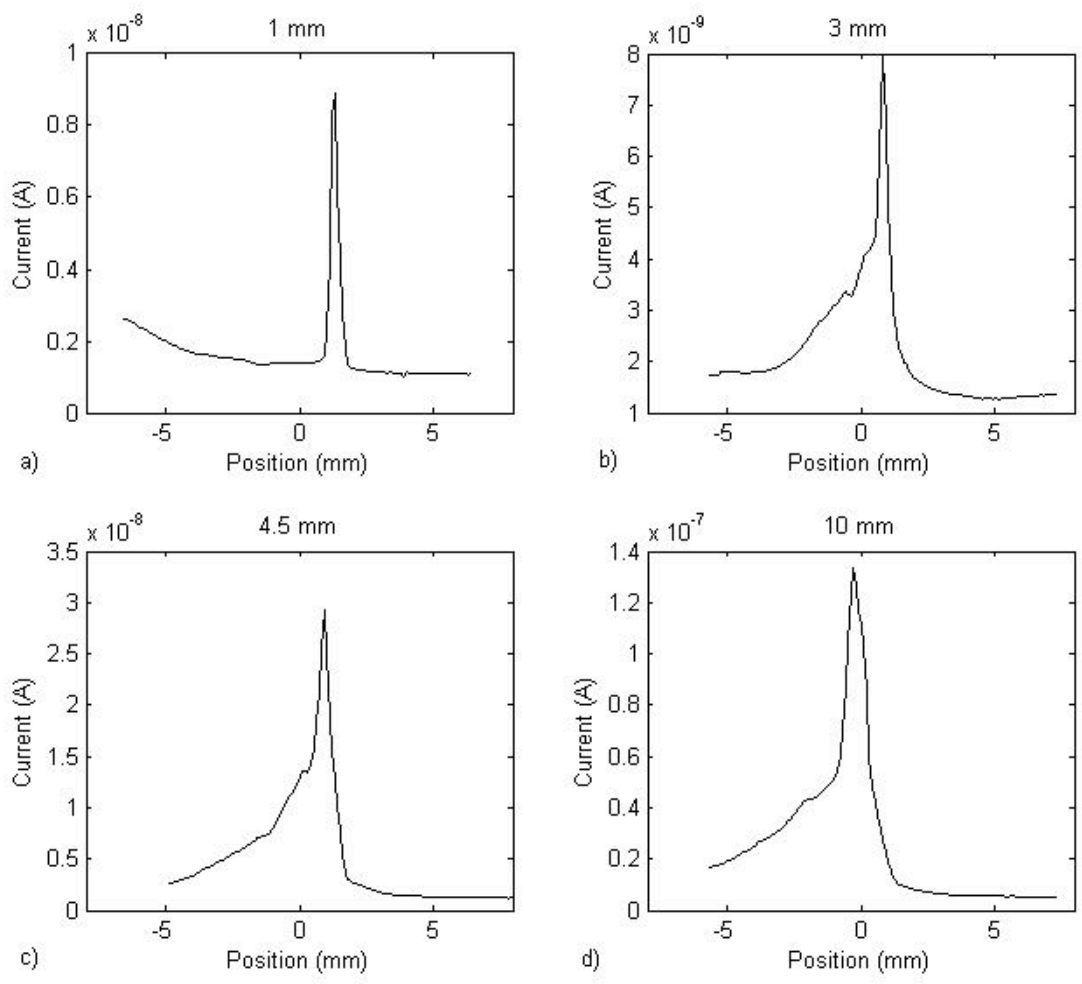

Figure 21a-d. Current profiles at $10 \mathrm{~V}$ at four heights above the magnet surface with no gas flow. 


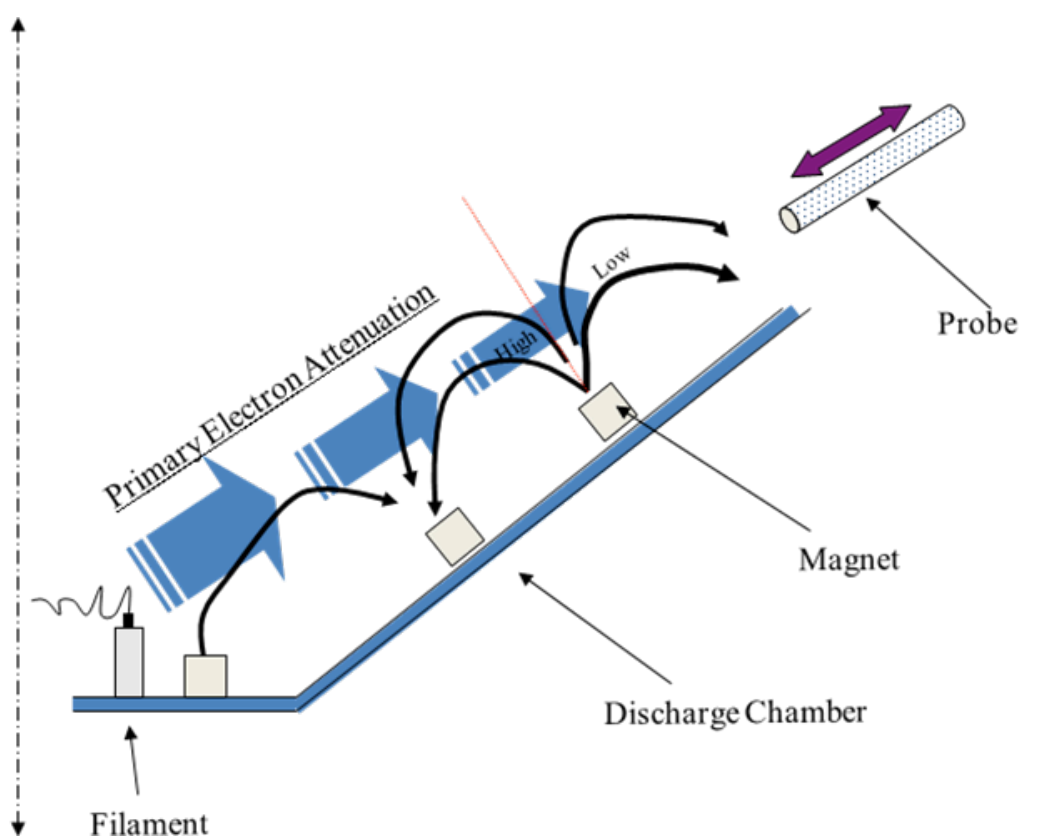

Figure 22. Schematic depiction of primary electron attenuation. Magnetic field lines are depicted by black arrows. The vertical centerline above the third magnet ring is depicted by the red line.

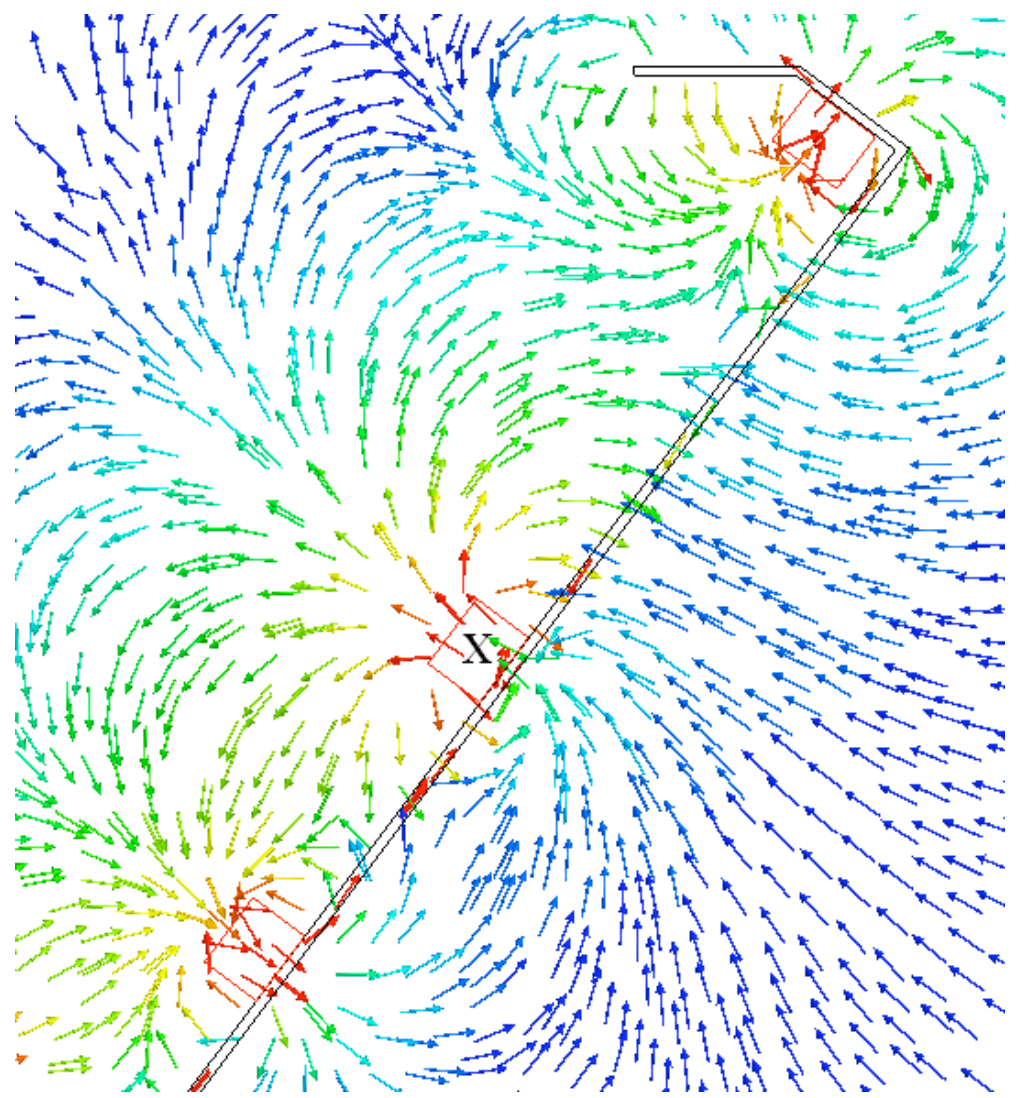

Figure 23. Vector plot of magnetic field demonstrating field asymmetry due to the positioning of the other magnet rings. The magnet ring surveyed by the cusp probe is indicated by an $X$. coupling with adjacent magnetic rings would tend to add to the distorted profile. These asymmetries are depicted in Figure 23 , a vector plot of the magnetic field near the third magnet ring.

Figure 24 shows the FWHM of the primary current data as a function of probe height above the magnet surface. The FWHM used in this case is associated with the narrow peak or "spike" shown in Figure 21. The narrow peak portion of the current line shape is associated with primaries injected along the axis of the cusp. The line shape of the narrow peaks was determined by deconvoluting the distorted profiles into a broad peak (associated with the shoulder) and a narrow peak. The FWHM was determined from a fit of this narrow peak. Interestingly, the loss width associated with the narrow peak varies initially slowly all the way down to $4.5 \mathrm{~mm}$ after which the widths narrow more quickly as the anode is approached. This observation reflects the shape of the cusp for primary electron transport. The loss width at the anode can also be inferred by extrapolating down to a probe height of zero. This was done by fitting the closest three data points to capture the rapid decrease in width. Again, as a rough approximation, a linear fit was used. The fit is shown in the figure. This crude fit suggests a loss width at the anode of approximately $0.23 \mathrm{~mm}$. This extrapolated value is consistent with twice the primary electron Larmor radius. The burn line widths acquired after extended low power operation with primaries only revealed weak impressions that were roughly $0.1 \mathrm{~mm}$ wide. The narrower than expected burn may be due to inadequate run time at these extremely low powers. Here, power density may not have been sufficient to yield a strong enough burn impression.

Figure 25 illustrates variations in the width of the narrow peak of the primary current profile as a 


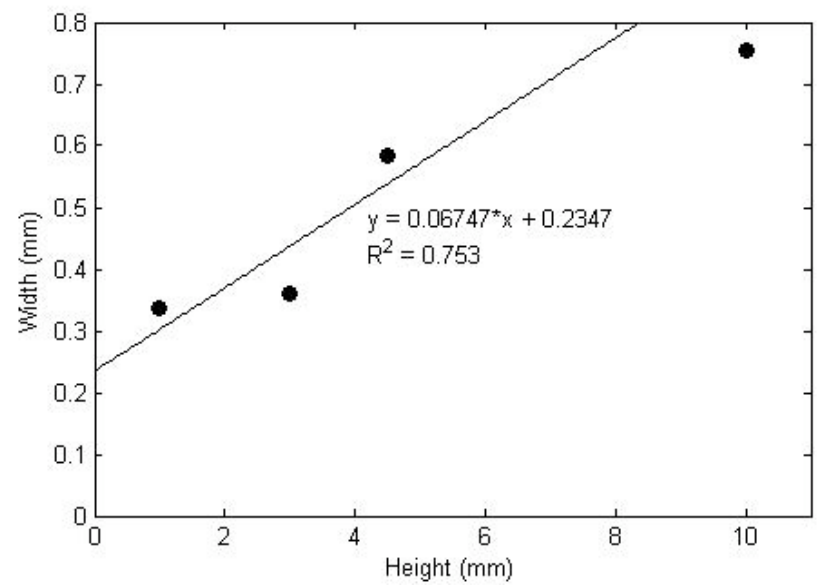

Figure 24. FWHM of narrow primary peak as a function of probe height.

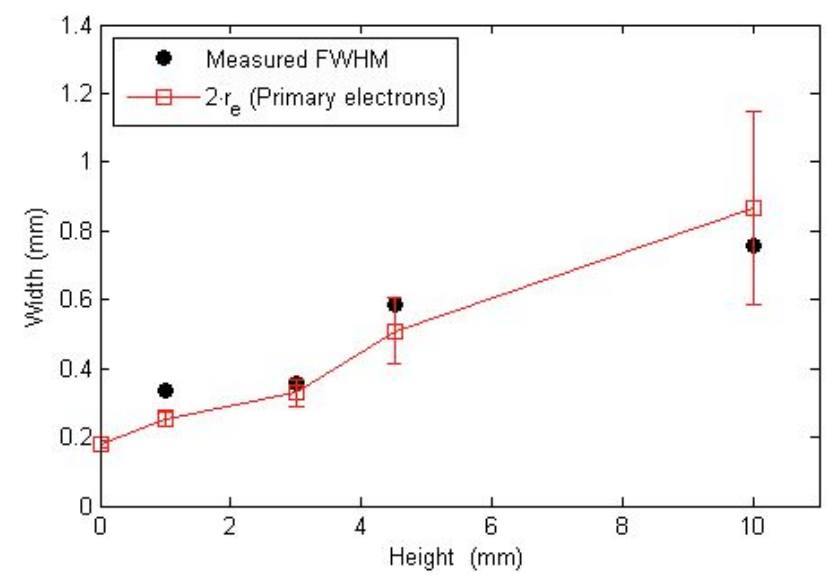

Figure 25. Comparison between measured FWHM of narrow primary peak and twice the primary electron Larmor radius. function of distance from the magnet surface. These are compared with the twice the primary electron Larmor radius. The profiles clearly agree as expected, with most of the measured data points falling within the error bars. Again, however, deviation is greatest at the largest height. Here the uncertainty in the assumed magnetic field is greatest.

\section{Concluding Remarks and Future Work}

Cusp collection plays a key role in determining discharge efficiency as well as stability. Plasma cusp measurements were assessed from a DC filament-driven multipole discharge operating at low gas utilization. Plasma loss width measurements were determined to be approximately equal to the hybrid radius. In the limit of zero input pressure, it was observed that loss widths approached twice the primary electron radius. Additionally, probe measurements seem to suggest a primary electron density gradient across the cusp at high heights above the magnet surface. This observation testifies to the directional nature of the injected primary electron flux. It was also found that the magnetic cusps have a collection efficiency such that all electrons that enter the cusp are not collected. This is believed to be associated with the acceptance loss cone of the magnetic cusps. Electrons that enter the cusps but are not collected circulate between magnetic rings. This behavior improves discharge efficiency. The study suggests that it is not simply the loss width at the anode surface alone that determines discharge efficiency; rather the transmission properties of the magnetic cusp play a key role. These properties are intimately related to the effective loss cone of the magnetic cusp. Future work will involve modeling and calculating the collection efficiency, as well as improved cusp probe measurements. Probe positioning will be improved by the use of a motorized positioning system, which will also allow for measurements at additional heights as well as a longer probe track. Future work will also involve investigation of cusp collection at higher gas utilization fractions $(>50 \%)$ as well as profiles in which a hollow cathode is the electron source. 


\section{References}

${ }^{1}$ Jones, R., “A source of error in cusp plasma leak measurements,” Plasma Physics, Vol. 21, 505, 1979.

${ }^{2}$ Pechacek, R.E. et al, "Measurement of plasma width in a ring cusp," Physics Review Letters, Vol. 45, No. 4, 256, 1980.

${ }^{3}$ Kozima, H., Kawamoto, S., and Tamagiwa, K., "On the leak width of line and point cusp magnetic fields," Physics Review Letters, Vol. 86A, No. 6,7, 373, 1981.

${ }^{4}$ Arakawa, Y., and Hamatani, C., "Reduction of Plasma loss to discharge chamber walls in a ring-cusp ion thruster," Journal of Propulsion and Power, Vol. 3, No. 1, 90, 1987.

${ }^{5}$ Morishita, T., Ogasawara, M., and Hatayama, A., Review of Scientific Instruments, Vol. 9, No. $2,968,1998$.

${ }^{6}$ Foster, J. and Patterson, M. "Internal Plasma Properties and Enhanced Performance of 8-Centimeter Ion Thruster Discharge," Journal of Propulsion and Power, Vol. 17, No. 2, 2001, pp. 428-432.

${ }^{7}$ Hershkowitz, N., Leung, K.N., and Romesser. "Plasma Leakage Through a Low-Beta Line Cusp," Physics Review Letters, Vol. 35, no. 5, 1975, pp. 277-280.

${ }^{8}$ Hershkowitz, N., Cho, M., Pruski, J., "Mechanical variation of plasma potential, electron temperature and plasma density," Plasma Sources Sci. Technol., Vol. 1, 87-93, 1992.

${ }^{9}$ Koch, C., and Matthieussent, G. "Collisonal diffusion of a plasma in a multipolar and picket fence device," Phys. Fluids, Vol. 26, No. 2, 1983, 545.

${ }^{10}$ Lieberman, M. and Lichtenberg, A. Principles of Plasma Discharges and Materials Processing, $2^{\text {nd }}$ ed., John Wiley \& Sons, New Jersey, 2005.

${ }^{11}$ M.S. Ioffe and E.E. Yushmanov, Nuclear Fusion Suppl., 1962, 177.

${ }^{12}$ Sadowsky, M. "Plasma confinement with spherical multipole magnetic field," Physics Letters 25A:695-696, 1967.

${ }^{13}$ Limpacher, R., and Mackenzie, K.R. "Magnetic multipole confinement of a magnetic field free plasma," Rev. Scientific Instruments 44:726-730, 1973.

${ }^{14}$ Sovey, J.S., "Improved Ion Containment Using a Ring-Cusp Ion Thruster," Journal of Spacecraft and Rockets, Vol. 21, No. 5, 1984, pp.488-495.

${ }^{15}$ Horrike, H., et al., "Cusp width and power flow study at high power magnetic multipole ion source," Phys. Fluids, Vol. 30, No. 10, 1987, 3268.

${ }^{16}$ Arakawa, Y., and Hamatani, C., "Analysis of Plasma Loss in a Ring Cusp Ion Thruster," Proceedings of the $19^{\text {th }}$ International Electric Propulsion Conference, AIAA Paper 87-1079, 1987.

${ }^{17}$ Goebel, D., Wirz, R. and Katz, I., "Analytical Ion Thruster Discharge Performance Model," Journal of Propulsion and Power, Vol. 23, No. 5, 1055-1067, 2007.

${ }^{18}$ Herskowitz, N. "How Langmuir Probes Work." In: O. Auciello and D.L. Flamm Editors, Plasma Diagnostic vol. 1 Academic Press, New York, 1989.

${ }^{19}$ Flamm, D., and Zeuner, M., "Spatially resolved measurements of plasma parameters in a broad-beam ion source," Surface and Coatings Technology, Vol. 116-119, 1999, pp. 1089-1092.

${ }^{20}$ Knorr, G., and Merlino, R.L., "The role of fast electrons for the confinement of plasma by magnetic cusps," Plasma Physics and Controlled Fusion, Vol. 26, No. 2, 443, 1984. 